by bit, the metal tube being pushed along the bone. The experiment shows that it is not necessary to sterilise the front surface of the bone by itself, for it is included in the sphere of activity of the steam which has sterilised the medullary cavity.

This method is only an attempt to extend the field of conservatism in the treatment of malignant tumours of bone.

\section{THE INFLUENCE OF THE X RAYS ON SOME CASES OF PERSISTENT SUPPURATION.}

By E. P. CUMBERBATCH, B.M. Oxon., M.R.C. P. Lond. MEDICAL OFFICER IN CHARGE OF THE ELECTRICAL DEPARTMENT, ST. BARTHOLOMEW'S HOSPITAL.

Two years ago $I$ tried the effect of the $\mathrm{X}$ rays upon a case of chronic suppuration of very long duration, which had resisted other forms of treatment, including surgical operation. The suppuration was arrested, the part healed, and the cure has lasted to the present time. Three other cases of a similar kind were then treated, and equally good results were obtained. During the past year other cases have been treated so as to investigate the action of the $\mathrm{X}$ rays upon chronic suppuration, and the results show that this form of treatment has an influence upon its progress, lessening it in some cases, and in others permanently arresting it. Many cases are still under treatment, but a short account will be given of the first four cases, as they have been kept under obserration for the longest time.

CASE 1.-A patient aged 22. A case of infective periostitis and prolonged suppuration of three years'duration. The past history showed that in 1904 the patient had had an abscess on her chin; it had healed spontaneously. In 1908 another abscess had appeared in the same situation. It "broke," and began to discharge. The discharge was small in amount but persistent. Early in 1910 a surgical operation was performed, and another in November of the same year, but after each the discharge soon reappeared. In February, 1911, the patient was admitted to hospital, the sinus was excised, the bone scrapod, and the wound stitched. In July it was necessary to repeat the operation, as the discharge had recurred. $A$ piece of dead bone was removed and some more was scraped away. In October a fifth operation was carried out, again without any lasting benefit. In December $\mathrm{X}$ ray treatment was commenced. There was then a sinus leading down to bone in the region of the symphysis of the lower jaw, with granulations extending for a quarter of an inch around and the outlying skin was adherent. One application of $\mathrm{X}$ rays was made in each period of three weeks, full doses being given (tint B, Sabouraud's method of measurement). After the fifth application the discharge greatly diminished; after the eighth it further diminished and became intermittent; and after the eleventh it ceased, and no more was noticed for three months. During this time the skin grew over the granulations, and the closed sinus and the site of the former suppuration was marked by a deep dimple with a small area of red skin at its base. At the end of the three months a slight discharge was again noticed and another application of $\mathrm{X}$ rays was made, and one more (the last given) a month later. The discharge ceased altogether, and no more was noticed during the succeeding 15 months. The patient was kept under observation during this time, and was last seen in February, 1914.

CASE 2.-A patient, aged 15. A case of chronic infective periostitis with chronic suppuration lasting six months. Past history: In December, 1911, a painful swelling appeared on the right side of the face over the lower jaw. The skin became red and then "broke," and a purulent discharge made its appearance and continued day after day without cessation. In June, 1912, the patient was taken to hospital. An operation was performed and a piece of dead bone was removed. The discharge soon reappeared. On July 23rd $\mathrm{X}$ ray treatment was commenced. There was then a firm swelling to be felt on the body of the lower jaw in the region of the angle, measuring about $1 \frac{1}{2}$ inches by $\frac{1}{2}$ inch. The skin overlying was red, and at its centre was a sinus from which pus was oozing. Smaller applications of $\mathrm{X}$ rays were given than in Case 1 , but at shorter intervals, one each week. After the second application the discharge lessened and became intermittent, and after the third the sinus closed and no more discharge was seen from that time onwards. Three more short applications were made, the last on Sept. 9th. In January, 1913, the swelling had diminished in size, and the skin overlying was slightly erythematous and a little keloid was present in it. In December the swelling could not be felt, and the keloid had disappeared. In March, 1914, a pink scar marked the site of the former suppuration and swelling.

CASE 3.-A patient aged 20. This was a case probably of hygroma which was subsequently infected. Past history: In 1908 the patient noticed a painless swelling on the back of the forearm just above the wrist. It very slowly enlarged. In 1911 the swelling was incised, and a "dark brown" fluid escaped. Soon after, a second swelling, similar to the first, appeared close to it, nearer the elbow. A third swelling also appeared over the wrist, but it disappeared spontaneously. The discharge from the first swelling persisted in spite of local treatment. On April 30th, 1913, X ray treatment was commenced. At that time there was a tense swelling on the posterior aspect of the forearm, about an inch in diameter slightly movable, and slightly raising the overlying skin, which was normal and not inflamed. This was the second swelling noticed by the patient. Immediately below was a sinus, from the opening of which was oozing a sero-purulent fluid. The bones of the forearm were $X$ rayed and found to be normal. Applications of $\mathrm{X}$ rays were made once a fortnight. After the second application the discharge consider ably diminished, and after the third it disappeared altogether. One more application was made. In January, 1914, the sinus was still closed. 'The swelling was still palpable, but it was considerably smaller.

C.ISE 4.-A patient aged 11. A case of tuberculous bursitis ; operation and subsequent chronic discharge. Past history: In January, 1913, the patient developed pain and swelling in front of one knee. In March an abscess in the prepatellar bursa was opened and the cavity scraped out. Tubercle bacilli were found. The wound healed by first intention, but later on it reopened at one point and pus continued to discharge slowly. In July, X ray treatment was commenced. At that time there was a thin sero. purulent discharge from a sinus leading to the prepatellar bursa. There was also clinical evidence of tuberculous infection of the knee-joint. The treatment was repeated once every fortnight. After the second application the discharge diminished, and after the third it disappeared. The patient was afterwards admitted to the Lord Mayor Treloar's Home at Alton. The disease became quiescent in the knee, but there was no more discharge from the former sinus (January, 1914).

These four cases showed chronic suppuration, small in amount, and in superficial situations. Case 1 received full applications of $\mathrm{X}$ rays at longer intervals; Case 2 receired small applications at short intervals; and Cases 3 and 4 received medium applications at intervals of medium length. The dosage was measured by Sabouraud's method. The most suitable technique with regard to dosage, penetration power of the rays, and the use of filters is difficult to decide, particularly as the modus operandi of the $\mathrm{X}$ rays in producing their effects on the tissues is not known. In arresting the process of suppuration the $X$ rars do not act only (if at all) by destroying the pyogenic organisms. In some experiments that were made to test the action of the $\mathrm{X}$ rays upon cultures of bacteria the pyogenic organisms were not destroyed. I am inclined to the belief that the $\mathrm{X}$ rays produce some local tissue reaction against 
the invading organisms, and at the same time some general reaction. In mainy of the cases that have been treated there has been considerable improvement in the general health, although in some of them the suppuration has not been arrested.

I am indebted to Mr. D'Arcy Power for permission to publish Case 1.

Upper Wimpole-street, $w$.

\section{attediral Socreties.}

\section{ROYAL SOCIETY OF MEDICINE.}

\section{CLINICAL SECTION.}

Exhibition of Clinical Cases and Pathological Specimens.

A MeETiNg of this section was held on May 8th, Mr. CharTers J. SYMonds, the President, being in the chair.

Dr. Clive RIviere showed a case of Spleno-myelogenic Leukæmia. It was that of a boy aged 12, who was admitted to hospital in December, 1913, with history of cough, breathlessness, pain in the right shoulder, and general weakness of one year's duration. He then was well nourished with a somewhat plethoric aspect. The spleen reached to the umbilicus; the liver $1 \mathrm{in}$. below the costal margin. Signs of enlarged thoracic glands were found mainly consisting of increase of oval interspinous duIness on the right side and impairment at the lung apex. A skiagram confirmed this. The urine contained much albumin. He had been treated by arsenic, $\mathrm{X}$ rays, and benzol. During the time the boy had been under observation he had felt well, had gained $6 \mathrm{lb}$. in weight, and his temperature had kept within normal limits. The pulse was accelerated, about 116. The liver had remained unaltered, but the spleen had increased in size both downwards and forwards. The area of lung impairment, incicative of enlarged chest glands, had also increased, and now extended between the first and ninth dorsal spines on the right side. The leucocytes had increased from 167,000 to 425,000 per c.mm.

Mr. SIDNEY A. BOYD showed a case illustrating the treatment by Splenectomy for Splenomegalic Cirrhosis with Ascites. The woman, aged 23, came under observation in November, 1913, for ascites, for which she had been in hospital in 1908. The notes of the case stated that the spleen was then somewhat enlarged. Paracentesis abdominis was performed several times, but the fluid quickly re-accumulated. Laparotomy was then performed, and the condition was thought to be due to tuberculous peritonitis. There were "some very suspicious caseous masses at the junction of the Fallopian tubes with the uterus, and partly on the broad ligaments. No definite tubercles could be seen elsewhere. The peritoneum looked very red, was much congested, and bled very freely." The abdomen was drained with a rubber tube for 24 hours, a large quantity of fluid escaping. She had made a good recovery from this operation, and remained in good health until October, 1913, when she noticed the abdomen again becoming distended. Upon examination in November, 1913, the patient was found to be a well-nourished young woman, not anæmic nor icteric, and with no signs of disease in the chest. The abdomen was greatly distended, the girth at the umbilicus being 36 in. No dilated veins were visible on the abdominal wall. The scar of the previous operation had stretched somewhat and bulged forwards. The distension was obviously due to ascites, and in the upper part of the abdomen an enlarged spleen could be felt. The liver was not palpable. Eight days later laparotomy was performed, the old scar being excised, and a large quantity of strawcoloured fluid escaped. There were no signs of tuberculosis on the parietal or visceral peritoneum, but a few adhesions were present between the coils of intestine. Except for some adhesions around the pelvic organs the latter appeared normal. The liver felt hard and irregular on the surface and smaller than normal. The spleen was considerably enlarged, and its convex surface was free from adhesions.
The parietal peritoneum was somewhat thickened. The incision was closed and healed well in seven days. The Wassermann reaction was negative. The ascites rapidly recurred after the operation. Paracentesis was performed and 15 pints of fluid drawn off. This was repeated, when 11 pints were drawn off. Splenectomy was then performed through a vertical incision at the outer edge of the left rectus. It was not possible to deliver the spleen through the wound on account of a broad band of adhesion from the posterior border to the posterior abdominal wall. The pedicle was therefore first tied off in sections and divided, and the adhesions posteriorly then dealt with. 'The branches of the splenic artery and vein were very large, but very little blood was lost. The liver was inspected and found to be in an extremely cirrhotic state, being not more than two-thirds the normal size, very hard and granular on the surface. There was practically no omentum present, only a few tags, or omentopexy would have been performed. The incision was closed in the usual way. The patient recovered well from the operation and her convalescence was smooth. The ascitic fluid collected again, but more slowly than before the operation. Three and a half weeks after the operation paracentesis was performed, $11 \frac{1}{2}$ pints being drawn off. Eleven days later the girth had increased from re-accumulation of fluid, but after this it gradually decreased and the fluid disappeared. It had not since re-collected, and the patient's general condition was good. Dr. A. G. Gibson had cultivated, after 18 days' incubation, a thick Gram-negative, non-motile bacillus which could not be continued in subculture. The remainder of the culture tubes remained sterile. This case was of interest both from the point of view of diagnosis and also from the effect of removal of the spleen. The case was obviously not one of Banti's disease (regarding Banti's disease as the terminal stage of splenic anæmia). There had been no anæmia, no leucopenia, no gastro-intestinal hæmorrhage. The spleen was known to have been enlarged in 1908, when laparotomy was performed for chronic peritonitis, probably of a tuberculous nature. Was it possible that the whole condition was due to tuberculosis - the splenomegaly, the hepatic cirrhosis, and the ascites? (Tarious French authors had raised the question of tuberculous hepatic cirrhosis.) The first laparotomy cured the ascites for five years. The second laparotomy had no effect on the ascites, which rapidly recurred. Removal of the spleen certainly had a direct effect upon it. The ascites could not have been due solely to perisplenitis, as the fluid re-accumulated at first after removal of the spleen and the abdomen had to be tapped. Nor would simple mechanical relief to the portal circulation, which splenectomy would entail, account for the result for the same reason. Removal of the spleen was not likely to influence the hepatic cirrhosis so favourably in a few weeks as to cause the ascites to disappear. On the other hand, if the spleen be regarded as a primary seat of infection by the organism grown from it, secondary to which a chronic peritonitis developed and also hepatic cirrhosis, this was an explanation which would satisfy all the conditions. This was the view which Mr. Boyd was inclined to take, and to consider the illness from which the patient suffered six years ago as an independent one, as far as the ascites was concerned. Time alone would tell whether the removal of the spleen had. arrested the progress of the cirrhosis, but from the point of view of relieving the ascites the operation had already justified itself.

Dr. ARThur F. Hertz showed three cases of Thomsen's Disease in members of the same family, two brothers aged 25 and 13, and a sister aged 16 . The degree of hypertrophy of the muscles was very marked,

Dr. F. PARKES WEBER showed a case of Progressive Vertebral Ankylosis ("Spondylose Rhizomélique"). The patient was a man aged 67. The whole of the vertebral column and the cranio-vertebral articulations appeared to be ankylosed in a position of kyphosis characteristic of such cases. The ribs apparently did not move with respiration, which was altogether diaphragmatic. He could open his mouth a little. The morement in both shoulders was much limited, more in the right than the left. The hip-joints were severely affected. The more distal joints of his limbs were free, excepting that he could not extend his kuees completely. In the semi-recumbent position he could feed himself with a spoon. He could walk about a little with the help of a stick. Some tremulousness of the hands, suggesting 